\title{
Feasibility and Performance of the Microwave Thermal Rocket Launcher
}

\author{
Kevin L.G. Parkin, Fred E.C. Culick \\ Division of Engineering and Applied Science, California Institute of Technology, \\ 1200 East California Boulevard, Pasadena, CA 91125, USA
}

\begin{abstract}
Beamed-energy launch concepts employing a microwave thermal thruster are feasible in principle, and microwave sources of sufficient power to launch tons into LEO already exist. Microwave thermal thrusters operate on an analogous principle to nuclear thermal thrusters, which have experimentally demonstrated specific impulses exceeding 850 seconds. Assuming such performance, simple application of the rocket equation suggests that payload fractions of $10 \%$ are possible for a single stage to orbit (SSTO) microwave thermal rocket. We present an SSTO concept employing a scaled X-33 aeroshell. The flat aeroshell underside is covered by a thin-layer microwave absorbent heat-exchanger that forms part of the thruster. During ascent, the heat-exchanger faces the microwave beam. A simple ascent trajectory analysis incorporating X-33 aerodynamic data predicts a $10 \%$ payload fraction for a 1 ton craft of this type. In contrast, the Saturn V had 3 non-reusable stages and achieved a payload fraction of $4 \%$.
\end{abstract}

\section{INTRODUCTION}

In the year 2003, payloads are launched into orbit the same way they were in 1963: by chemical rockets. Traditional expendable multistage rockets usually achieve payload fractions of less than $5 \%$. As described by the rocket equation, this is due partly to the structural limits of existing materials, and partly to the limited specific impulse $\left(\mathrm{I}_{\mathrm{sp}}\right)$ of chemical propellants, which have reached a practical limit of 480 seconds. The structural economies made to preserve these minute payload fractions result in fragile rockets that are expensive to build. Despite 40 years of incremental rocket development, materials improvements have proven ineffective, novel propellants impractical, reliability is still variable, and the cost of launch has remained around $\$ 5000$ per kilogram of delivered payload.

The microwave thermal rocket, shown in figure 1, is a reusable single stage vehicle that can afford the mass penalty of a robust, low cost, construction because it a uses a high performance microwave thermal propulsion system with double the $I_{s p}$ of conventional rockets. It is premature to quantify the reduction in transportation costs that these simplifications will bring, but clearly the cost reduction could be dramatic, completely transforming the economics of launch, and the way that we use and explore space. 


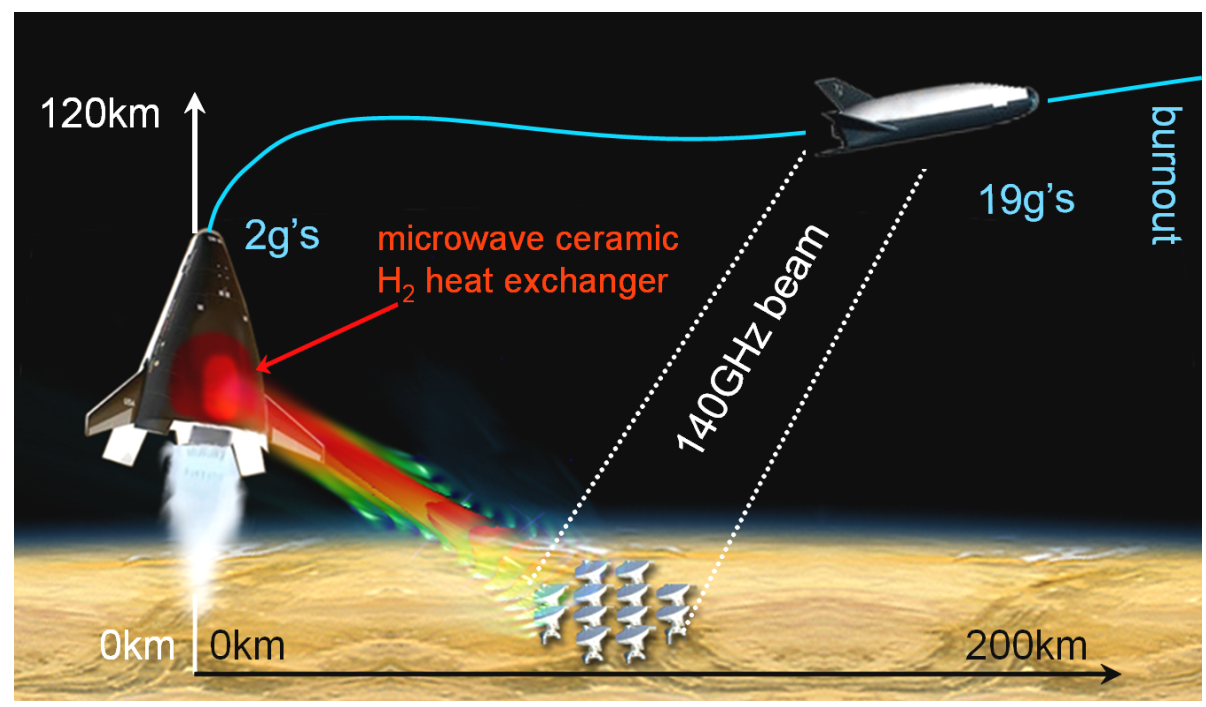

FIGURE 1. The Microwave Thermal Rocket (MTR)

The microwave thermal thruster is the key enabling component of the microwave thermal rocket. It is fundamentally a hydrogen heat-exchanger, the feasibly and performance of which we examine in an accompanying paper [1]. Hydrogen heatexchanger propulsion is not new [8,9]. Nuclear thermal thrusters operate on the hydrogen heat-exchange principle using neutrons as an energy source, rather than microwaves. From the 1950 s to the 1970 s a series of over 30 nuclear thermal thruster tests, conducted as part of the KIWI and NERVA programs, experimentally demonstrated that the hydrogen heat-exchanger approach can produce an $\mathrm{I}_{\mathrm{sp}}$ of 850 seconds. Furthermore, demonstrating that it can do so at power levels exceeding $1 \mathrm{GW}$ (for high thrust), and for durations of over an hour. But the microwave thermal thruster geometry is different; unlike nuclear rockets, which contain a neutron gas in a cylindrical heat exchange geometry using neutron reflective walls, the microwave rocket intersects a transatmospheric microwave beam in the most efficient way possible; upon the flat microwave-absorbent underside of a lifting body aeroshell.

We have calculated the microwave thermal rocket ascent trajectory, shown approximately in figure 1 , for a 1 ton, $275 \mathrm{MW}$ vehicle that inserts a $100 \mathrm{~kg}$ payload into a $1100 \mathrm{~km}$ circular orbit. To our knowledge this is the first quantitative microwave beamed-energy ascent trajectory, and demonstrates that a microwave thermal rocket can indeed achieve low Earth orbit (LEO) with a 10\% payload fraction, as predicted by the rocket equation. Aerodynamic data obtained from SR-71 flight tests of the X-33 aeroshell are incorporated into the ascent trajectory simulation, and the propulsion system is modeled using simple ideal relations between power input, specific impulse, and thrust. The ascent acceleration varies from 2 to $19 \mathrm{~g}$ 's in order to stay within range $(150 \mathrm{~km})$ of the microwave beam source in boost phase. 


\section{FEASIBILITY}

\section{Trans-Atmospheric Microwave Beam Propagation}

Beamed-energy concepts are limited to frequencies at which the atmosphere is transparent. Near total absorption by $\mathrm{H}_{2} \mathrm{O}$ in a large portion of the far infrared spectrum divides viable beamed-energy concepts into two categories; laser and microwave. Beaming energy sufficient to propel a ton into LEO requires $100 \mathrm{MW}+$ of energy transmission through the atmosphere. Microwaves have two main advantages: First, at microwave wavelengths, atmospheric turbulence is not the major problem it is with lasers. Second, commercially available microwave sources are already capable of generating this level power output; whereas today's most powerful lasers are still two orders of magnitude weaker.

The advent of submillimeter astronomy has highlighted the existence of locations with particularly low atmospheric water content, opening up new microwave transmission windows between $35-300 \mathrm{GHz}$ and sometimes beyond. Since the atmospheric scale height of water vapor is only 1-2 km, sites such as the Caltech Submillimeter Observatory (CSO) on Mauna Kea are at high altitude, where atmospheric water vapor levels permit transmission above $250 \mathrm{GHz}$, shown in figure 2. Atmospheric propagation conditions are still better in parts of the Chilean Atacama desert, and Antarctica. Ongoing site surveys and millimeter wavelength projects, such as the CARMA array in Eastern California, may reveal suitable locations for a beam facility on the US mainland.
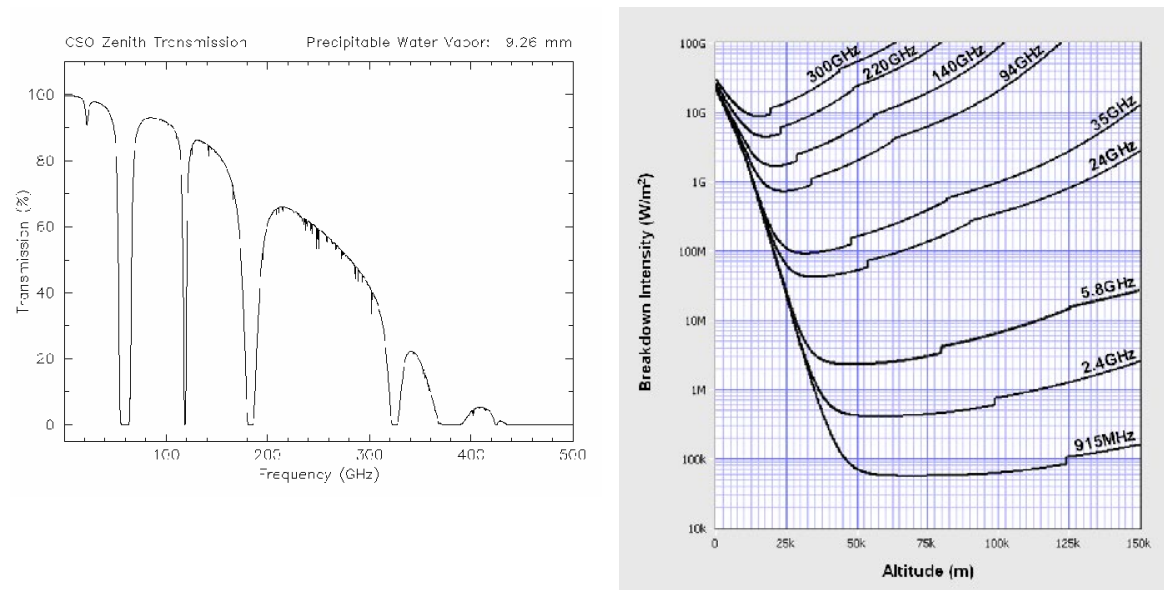

FIGURE 2. CSO atmospheric transmission from Mauna Kea calculated for high water content using [2]. Typical atmospheric water content (PWV) is $1 \mathrm{~mm}$. $9 \mathrm{~mm} \mathrm{PWV}$ is particularly poor FIGURE 3. Atmospheric breakdown intensity by altitude and frequency. Calculated using the semi-empirical model given by Liu et. Al. [3] atmospheric propagation conditions. 
Using a phased array source, a high intensity 3 meter diameter microwave spot can be projected onto the underside of a launcher over $100 \mathrm{~km}$ away. High frequency operation above $100 \mathrm{GHz}$ is preferable because the array area needed decreases substantially as beam frequency increases. The feasibility and design of a $30 \mathrm{MW}$, $245 \mathrm{GHz}$, ground-based beam facility using an array of parabolic dishes has already been examined in papers by Myrabo, Benford, and Dickinson [4,5]. Since the publication of these papers in the mid-1990s, the time-average power output of the gyrotron sources used has significantly increased, in addition to the 6 order of magnitude increase in the preceding 30 years. At present levels, the power output of $3001 \mathrm{MW}$ gyrotron sources operating at $140 \mathrm{GHz}$ is sufficient to propel a 1 ton craft into LEO.

Microwave frequency determines the maximum beam energy density via the constraint of atmospheric breakdown. Atmospheric breakdown occurs more easily at low frequencies, ionizing air into a plasma that can distort and reflect the incoming beam. We have calculated the continuous wave $(\mathrm{CW})$ atmospheric breakdown intensity, shown in figure 3. This chart shows that a $300 \mathrm{GHz}$ beam can achieve 1000 times the power density of a $3 \mathrm{GHz}$ beam, assuming that it is constrained at the altitude of minimum breakdown intensity.

\section{The Rocket Equation}

The rocket equation can be used in conjunction with existing launcher data to estimate the feasibility of a microwave thermal rocket. Following the nomenclature of Pisacane \& Moore [6] the payload mass fraction $\left(\mathrm{r}_{1}\right)$ and structural mass fraction $\left(\mathrm{r}_{\mathrm{s}}\right)$ is defined by

$$
r_{l} \equiv \frac{m_{l}}{m_{i}}, \quad r_{s} \equiv \frac{m_{s}}{m_{s}+m_{p}}
$$

where $\mathrm{m}_{\mathrm{i}}, \mathrm{m}_{\mathrm{l}}, \mathrm{m}_{\mathrm{s}}$, and $\mathrm{m}_{\mathrm{p}}$ are the initial, payload, structural, and propellant masses respectively. Assuming a single stage and neglecting drag, the rocket equation can be written

$$
r_{l}=\frac{e^{-\Delta v / g I_{s p}}-r_{s}}{1-r_{s}}
$$

Choosing $\Delta \mathrm{v}=9.6 \mathrm{~km} / \mathrm{s}$, a typical value required to reach LEO, equation (2) is plotted in figure 4 to graphically depict the tradeoff between payload fraction, structural efficiency, and propulsive performance for an SSTO launch concept. Where possible, data from other launch concepts has been used to bound the expected performance of a microwave thermal rocket. For multi-stage launchers, the data point is interpreted as an 'SSTO equivalent performance', usually deduced from the payload and structural mass fractions, since any two unknowns in (2) can be used to place a point on the figure. In all cases, the $\mathrm{I}_{\mathrm{sp}}$ represents an averaged value over the entire ascent. 


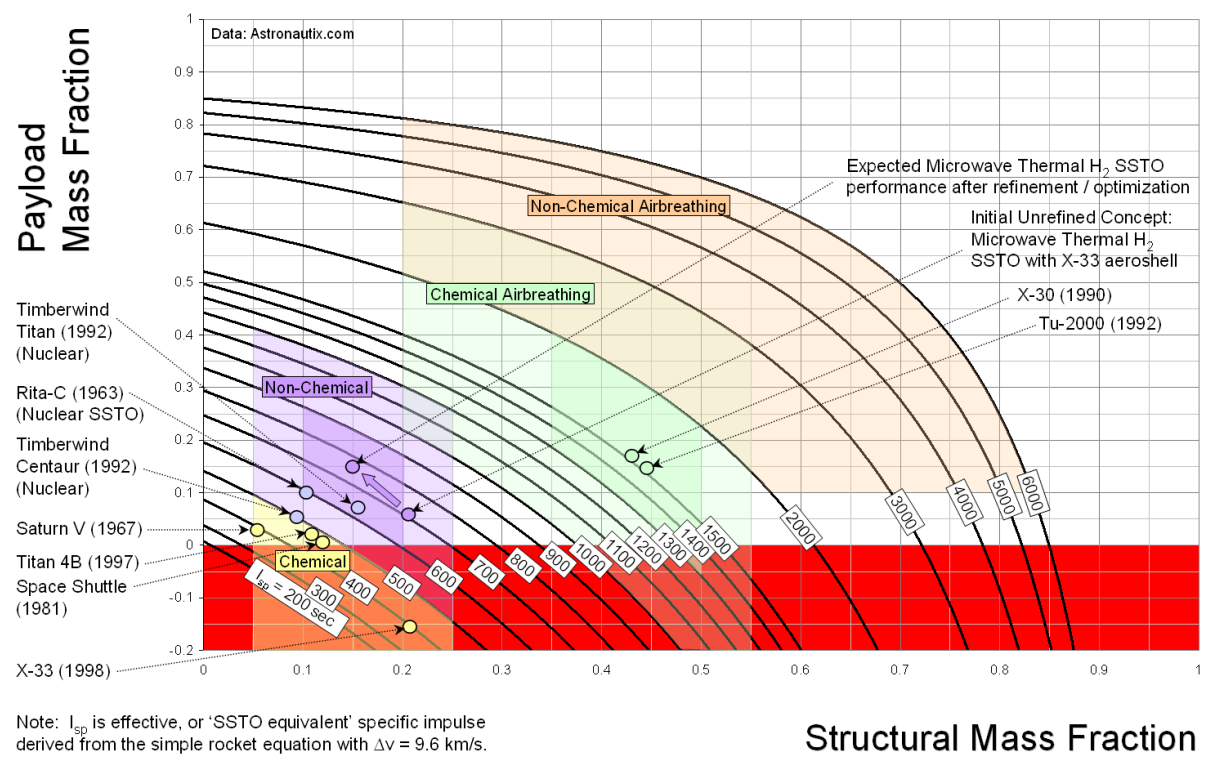

FIGURE 4. SSTO equivalent propulsive, structural, and payload performance of launchers.

Past studies of nuclear launch concepts, particularly RITA-C and the X-33 concept, provide bounds on the likely balance between propulsion, structure, and payload for microwave thermal rockets. Assuming the structural inefficiency of the X-33 combined with an average propulsive performance $\left(\mathrm{I}_{\mathrm{sp}}\right)$ of 720 seconds, we can graphically predict a 5\%-15\% payload fraction for the microwave thermal rocket.

\section{Aeroshell and Propulsion}

Like the Space Shuttle and X-33 concept, the microwave thermal rocket uses a lifting body aeroshell. The X-33 aeroshell was intended to be a great improvement on the shuttle aeroshell, giving a much greater cross-range. Though no chemicallypropelled SSTO vehicle appears to have the performance to use it, it turns out to be ideal for a microwave SSTO launcher due to its wide, flat underside, shown in figure 5. This underside serves as the heat-exchanger component of the microwave thermal thruster, facing roughly toward the microwave beam during powered ascent. Since lifting body aeroshells have been studied, built, and tested, we regard them as feasible.

The heat-exchanger also functions as part of the reentry heat shield. Further investigation of the microwave thermal thruster [1] has identified silicon carbide as a possible construction material, in part because of its microwave absorbent properties. Silicon carbide is already used as part of the space shuttle reentry heat shielding due to its excellent oxidation resistance at high temperatures. However, the most convincing argument for feasibility of the microwave thermal thruster will be a demonstration. 


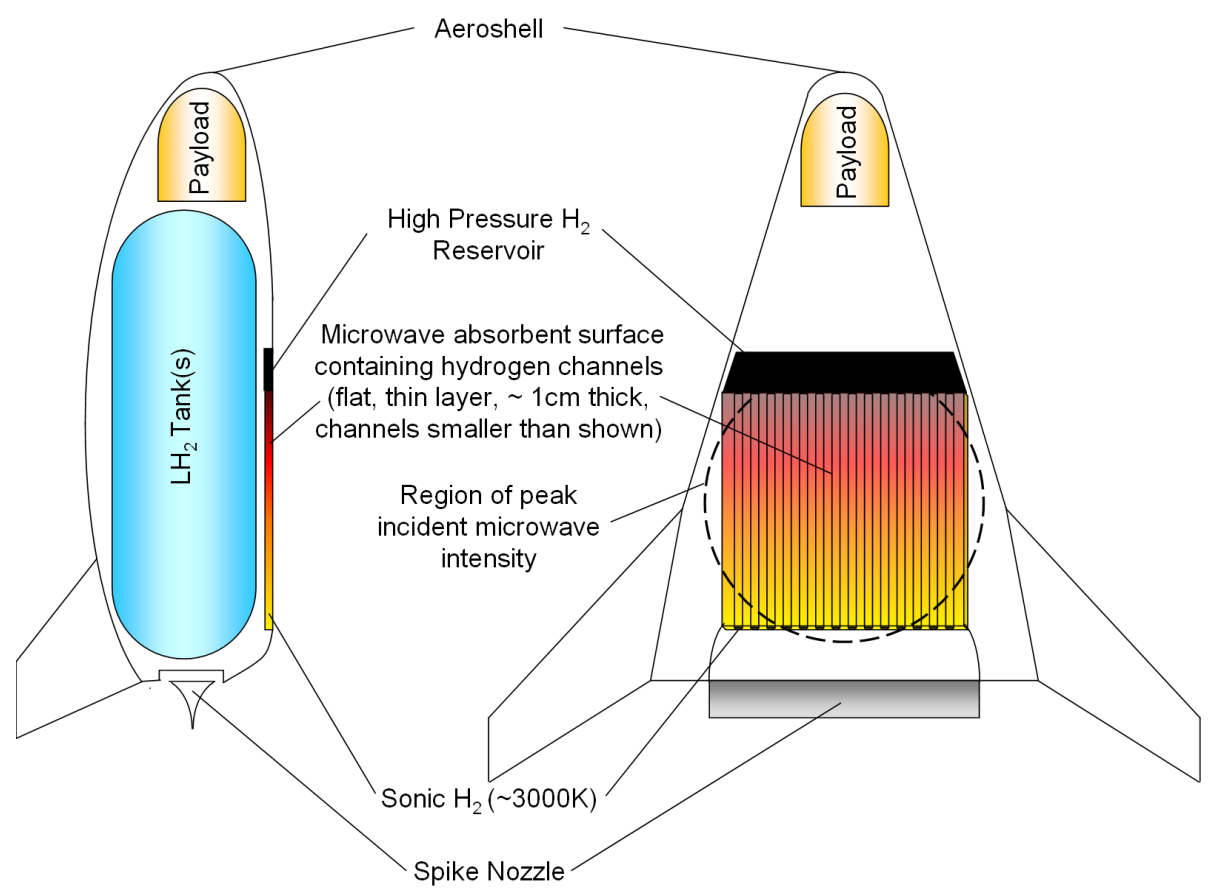

FIGURE 5. A lifting body launcher configuration with microwave thermal thruster forming part of the vehicle underside.

A spike nozzle is used for optimum expansion of the heat-exchanger propellant exhaust at all altitudes. Again, spike nozzles have been well-studied. In addition to using an $\mathrm{LH}_{2}$ propellant, which has a very low specific density; ammonia, alcohol, or other propellants can be used to reduce the necessary volume of the launcher, at the expense of somewhat reduced $\mathrm{I}_{\mathrm{sp}}$.

\section{PERFORMANCE}

\section{Ascent Trajectory Model}

While the rocket equation analysis indicates the feasibility of the microwave thermal rocket concept, we use an ascent trajectory analysis to confirm this finding by modeling the performance of a particular design, in this case a one ton launcher with X-33 type aeroshell. Ascent trajectory equations are derived from a non-inertial control volume analysis in the radial coordinate system shown in figure 6 . Equation (3) is the continuity equation and $(4,5)$ are momentum conservation equations along the $(\hat{r}, \hat{\theta})$ unit vectors respectively. Unlike fixed Cartesian unit vectors, the radial ones have nonzero time derivatives. 


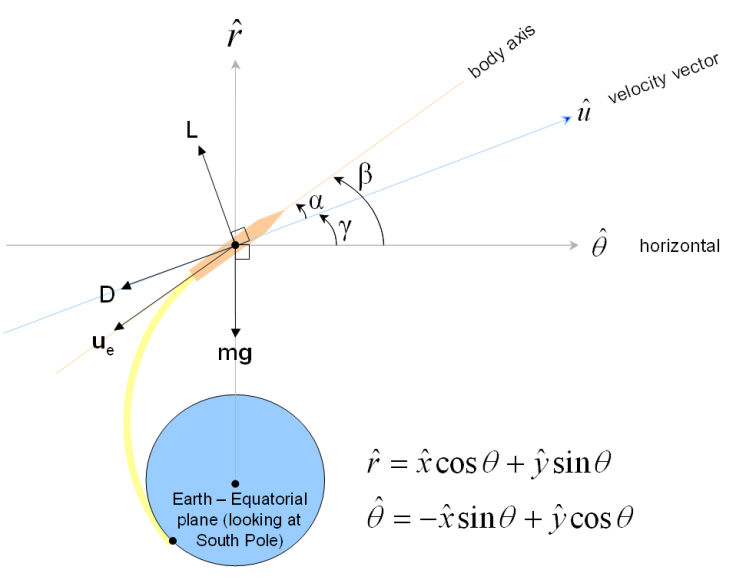

FIGURE 6. The ascent trajectory coordinate system.

$$
\begin{array}{cc} 
& \dot{m}=-\dot{m}_{p} \\
\hat{r}: & m\left(\ddot{r}-r \dot{\theta}^{2}\right)=T \sin \beta-D \sin \gamma+L \cos \gamma-m \mu / r^{2} \\
\hat{\theta}: & m(2 \dot{r} \dot{\theta}+r \ddot{\theta})=T \cos \beta-D \cos \gamma-L \sin \gamma
\end{array}
$$

$(m, r, \theta)$ are the unknowns to be integrated, representing mass, radius, and angular displacement respectively. $\beta$ is a trajectory steering angle that we specify. Thrust $\mathrm{T}$ and propellant mass flow rate $\dot{m}_{p}$ are calculated from the propulsion model, and lift $\mathrm{L}$ and drag $\mathrm{D}$ are calculated from the aerodynamic model. In the limit that thrust, drag, and lift tend to zero, $(4,5)$ reduce to Kepler's equation, as we would expect. $\mu=3.9863 \times 10^{14} \mathrm{~m}^{3} \mathrm{~s}^{-2}$ for Earth, and $\gamma$ is the angle between the horizontal and velocity vector, found at any given instant from

$$
\gamma=\tan ^{-1}(\dot{r}, r \dot{\theta}), \quad u=\sqrt{\dot{r}+(r \dot{\theta})^{2}}
$$

$\mathrm{u}$ is velocity in the direction of motion, which is needed to calculate lift and drag:

$$
D=\rho u^{2} A C_{D} / 2, \quad L=\rho u^{2} A C_{L} / 2
$$

Atmospheric density $\rho(\mathrm{r})$ is calculated from a 1976 standard atmosphere and $\mathrm{A}$ is the aeroshell frontal area, obtained by scaling the aeroshell geometry based on $\mathrm{LH}_{2}$ density. The lift and drag coefficients are obtained from the launcher aerodynamic model. In this case, a zero angle of attack is assumed for the atmospheric portion of flight, and the coefficient of drag is approximated using Mach 0.6 to 1.8 drag data [7] from SR-71 testing of the LASRE X-33 aeroshell model:

$$
C_{D_{0}}=\left\{\begin{array}{cc}
0.3 & M<0.9 \\
6 M-5.1 & 0.9 \leq M<1 \\
-1.1667 M+2.0667 & 1 \leq M<1.3 \\
0.55 & 1.3 \leq M
\end{array}\right.
$$


In the propulsion model, a throttle factor $\eta$ is specified for the trajectory

$$
T=g \dot{m}_{p} I_{s p}, \quad P_{j}=\dot{m}_{p}\left(g I_{s p}\right)^{2} / 2, \quad \dot{m}_{p}=\eta\left(\dot{m}_{p}\right)_{\max }
$$

where $g=9.81 m \cdot s^{-2}, \mathrm{P}_{\mathrm{j}}$ is jet power, and $\left(\dot{m}_{p}\right)_{\max }$ is a chosen maximum propellant mass flow rate. Finally, the variation of $I_{s p}$ with altitude is

$$
I_{s p}=\frac{1}{g} \sqrt{2 c_{p}\left(T_{t}^{*}\right) T_{t}^{*}\left(1-\frac{p_{a}}{p_{t}^{*}}\right)^{\gamma-1 / \gamma}}
$$

where $T_{t}^{*}, P_{t}^{*}$ are wall temperature and total pressure at the channel exit respectively. Since the sonic point is the highest temperature point in the thruster, $T_{t}^{*}$ is chosen to be $2800 \mathrm{~K}$, and $P_{t}^{*}$ is conservatively chosen to be $20 \mathrm{~atm} . \quad P_{a}$ is the ambient atmospheric pressure, obtained from the atmospheric model at any given altitude.

\section{Vehicle Scaling}

Vehicle scaling is based upon the dimensions of the LASRE model [7], whose shape is approximated to a triangular pie slice. Based on an $\mathrm{LH}_{2}$ density of $70 \mathrm{~kg} / \mathrm{m}^{3}$ and propellant mass requirement of $720 \mathrm{~kg}$, the dimensions of the LASRE model are scaled up by a factor of 1.7 to $\mathrm{L}=6.0 \mathrm{~m}, \mathrm{~W}=5.0 \mathrm{~m}, \mathrm{H}=1.3 \mathrm{~m}$, giving a total volume of $19.5 \mathrm{~m}^{3}$ and frontal area of $6.5 \mathrm{~m}^{2}$. Volumetrically, this is larger than needed for the fuel tanks, which only occupy $53 \%$ of the volume at this scale. The value for frontal area is used in the ascent trajectory drag calculations.

\section{Results}

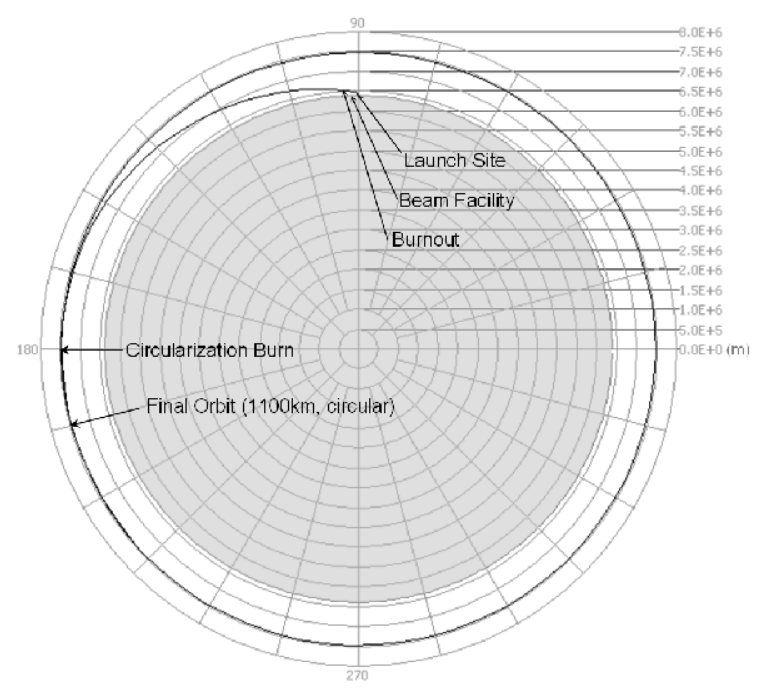

FIGURE 7. Whole-Earth view of the launcher ascent trajectory. 

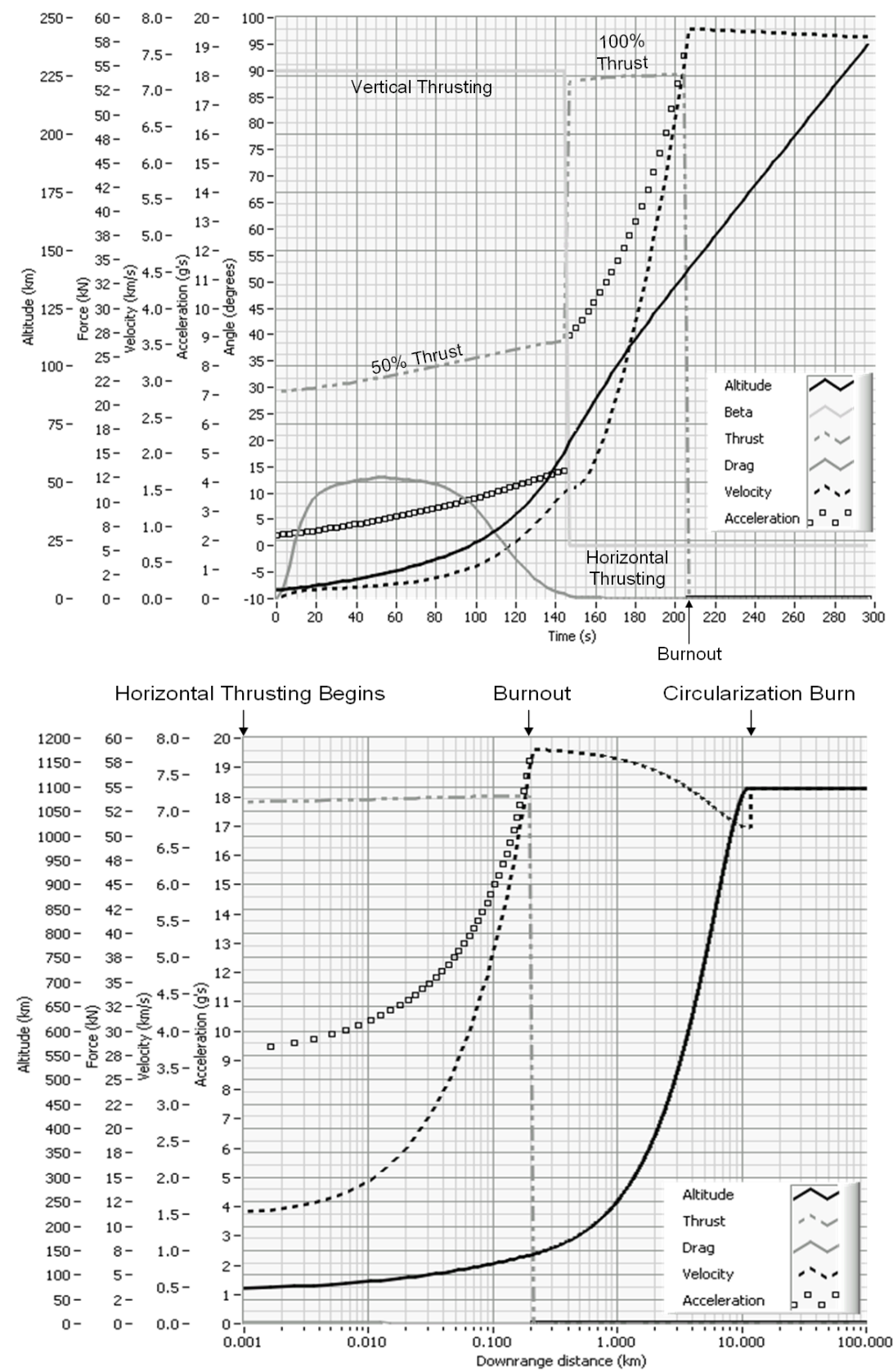

FIGURE 8. (Top) Profile of the ascent trajectory by time. (Bottom) Profile of the ascent by downrange distance. Note that this diagram does not capture the vertical portion of ascent. 
Taking into account atmospheric drag and gravity losses, the predicted payload is $10 \%$ for the baseline microsatellite launcher, characterized by:

- 1 ton vehicle wet mass: $100 \mathrm{~kg}$ payload, $180 \mathrm{~kg}$ structure, $720 \mathrm{~kg} \mathrm{\textrm {LH } _ { 2 }}$

- 6 meter vehicle length. $5 \mathrm{~m}$ width at base excluding wings. 3 meter diameter beam footprint (on vehicle), $150 \mathrm{~km}$ maximum required beam range

- $100 \mathrm{~kg}$ payload to $1100 \mathrm{~km}$ circular orbit (with circularization burn)

- $275 \mathrm{MW}$ jet power for $54 \mathrm{kN}$ of thrust at an $\mathrm{I}_{\mathrm{sp}}$ of 775-1030 seconds

- 210 second ascent from ground to 'burnout', with $\mathrm{H}_{2}$ Mass flow rate of $5 \mathrm{~kg} / \mathrm{s}$ at $100 \%$ throttle

- 2 g's initial acceleration, 19 g's peak.

- $3 \mathrm{~m} \times 3 \mathrm{~m}$ heat exchanger with average power density of $30 \mathrm{MW} / \mathrm{m}^{2}$

- Average microwave beam intensity 65 times lower than $140 \mathrm{GHz}$ atmospheric breakdown threshold at worst-case altitude

- Delta-V budget (integrated along direction of motion): Thrust $+11044 \mathrm{~m} / \mathrm{s}$, Drag $-1513 \mathrm{~m} / \mathrm{s}$, gravity $-2753 \mathrm{~m} / \mathrm{s}$, circularization burn $+523 \mathrm{~m} / \mathrm{s}$. Total: $7031 \mathrm{~m} / \mathrm{s}$ (equal to $1100 \mathrm{~km}$ circular orbit velocity).

On a planetary scale, the ascent trajectory is a spatially small maneuver that is just resolvable at the top of figure 7. A burn of $523 \mathrm{~m} / \mathrm{s}$ is performed at the transfer trajectory apoapsis, inserting the whole craft into of $1100 \mathrm{~km}$ circular orbit. This delta- $\mathrm{V}$ is similar to that imparted by rotation of the Earth, and both are unaccounted for in the vehicle propellant budget. Depending on the mission, it may be sufficient to circularize only the payload and allow the craft to re-enter, saving the propellant mass of an orbital maneuvering system that may use conventional thrusters.

The ascent trajectory itself consists of two segments. In the first segment, seen at the top left of figure 8 , the vehicle is steered vertically $\left(\beta=90^{\circ}\right)$ at $50 \%$ throttle to minimize drag losses as it ascends through the atmosphere. When a transition altitude of $65 \mathrm{~km}$ is reached, the second segment of ascent begins as the craft levels off, and thrusts horizontally $\left(\beta=0^{\circ}\right)$ at $100 \%$ throttle. The extreme acceleration of $9-19 \mathrm{~g}$ 's during this segment of the ascent raises the craft velocity from $1.5 \mathrm{~km} / \mathrm{s}$ to $8 \mathrm{~km} / \mathrm{s}$ in only 60 seconds. Such acceleration enables the vehicle to achieve orbital velocity within $150 \mathrm{~km}$ of the beam source, which is the maximum beam range, corresponding to $200 \mathrm{~km}$ downrange of the launch point, as seen at the bottom of figure 8 .

\section{CONCLUSIONS}

The microwave ascent trajectory has highlighted two significant characteristics of microwave thermal launchers: First, that the microwave thermal thruster needs to operate with an off-normal beam incidence angle up to $\pm 45^{\circ}$ (and we show in [1] that the off-normal performance is indeed sufficient). Second, that the power needed to launch a 1 ton vehicle whose burnout occurs within $150 \mathrm{~km}$ of the beam source is about three times greater than needed to launch the same vehicle with an unconstrained beam range. In this sense, beam range is traded against beam power. 
Most importantly, we have demonstrated that the microwave thermal launcher could achieve high payload performance with a feasible ascent trajectory. We have pointed out that suitable gyrotron microwave sources already exist, referred to a phased array design to generate the required beam, and shown that the $140 \mathrm{GHz}$ microwave beam should propagate through the atmosphere. In [1] we have given a rudimentary microwave thermal thruster design, and calculated that the performance should be sufficient for the 1 ton launch concept presented here.

The microwave thermal approach aims to solve an important problem of our time; the launch problem. The merit of this approach is a function only of the principles it employs, not the perceived insolubility of the launch problem or how unimaginable any aspect sounds. Free from the significant theoretical and engineering impediments of hypersonic airbreathing launch, we conclude that the technical obstacles to building a microwave thermal launcher in the near-term are surprisingly low.

\section{ACKNOWLEDGMENTS}

The author is grateful to Prof. Albert Ratner for numerous helpful discussions and to the Caltech President's Fund for supporting this work under grant PF-495 / NASA contract NAS7-1407.

\section{REFERENCES}

1. Parkin, K.L.G, DiDomenico, L.D., and Culick, F.E.C., "The Microwave Thermal Thruster Concept" in Proc. Second International Symposium on Beamed Energy Propulsion-2003, New York: American Institute of Physics.

2. Lis, D., CSO Atmospheric Transmission Interactive Plotter, Pasadena: California Institute of Technology (http://www.submm.caltech.edu/cso/weather/atplot.shtml).

3. Liu, G., Liu, J., Huang, W., Zhou, J., and Song, X., SPIE 3158, 238-248 (1997).

4. Myrabo, L., and Benford, J., "Propulsion of Small Launch Vehicles using High Power Millimeter Waves" in Proc. OE/LASE'94 Conference, Los Angeles, CA, January 22-29 (1994)

5. Benford, J., and Dickinson, R., Intense Microwave Pulses III SPIE 2557, 179 (1995).

6. Pisacane, V.L., "Force-Free Rocket Motion," in Fundamentals of Space Systems, edited by V.L. Pisacane and R.C. Moore, New York: Oxford University Press, 1994, pp. 189-195.

7. Whitmore, S.A., and Moes, T.R., "A Base Drag Reduction Experiment on the X-33 Linear Aerospike SR-71 Experiment (LASRE) Flight Program” Presented at the 37th AIAA Aerospace Sciences meeting and Exhibit, January 11-14, 1999, Reno, Nevada. AIAA 99-0277.

8. Kare, J.T., "Near-Term Laser Launch Capability: The Heat Exchanger Thruster" in Proc. First International Symposium on Beamed Energy Propulsion-2003, New York: American Institute of Physics.

9. "Nuclear Rockets" in Propulsion Techniques, edited by P.J. Turchi, Reston, Virginia: AIAA Press, 1998, pp. 181-273. 